\title{
Importance of balance diet to gain the quality of benefits for human life
}

\section{Opinion}

A balanced diet should contain all the below mentioned components to sustain healthy human life. The diet should be full of nutrients so that human beings can perform daily tasks energetically. Our balanced diets should contain $15-20 \%$ of calories from protein, $25-35 \%$ of calories from fat and $50-55 \%$ of calories from carbohydrates. One should prefer complex carbohydrates in the form of fruits, vegetables and grains over simple carbohydrates (white flour and sugar). Our body needs different nutrients to function properly as such our diets should contain all nutrients to provide the body with every nutrient needed by it.

In a balanced diet, intake of the five food groups including vegetables \& fruits, grains, milk, meat, oils and sugars should be adequately consumed. According to the international guidelines, our daily intake from vegetable group should be 2-3 servings including vegetables of different colors because each has different pigments and different advantages. One serving of vegetable equals to 1 cup raw vegetable or $1 / 2$ cup cooked vegetable or 1 cup salad. Besides this, fruit intake should be 2-3 servings including mainly fresh fruits. One serving of fruit includes 1 banana or 1 apple or 1 orange or $1 / 2$ glass fruit juice. Average milk intake for children and adults is recommended to 1-2 cup. Avoid full-fat milk and regular use of low fat milk and its products is highly beneficial. One serving size of milk equals 1 glass of milk or 1 cup yogurt. Average meat intake is 2 servings which include lean meat, chicken or fish. One serving of meat group equals 2-3 small chicken/fish pieces or 1 egg. Average grain intake is 6-11 servings, including $100 \%$ whole grain products rather than refined ones. One serving of grain equals $1 / 2$ chapatti or $1 / 2$ cup cooked rice or 1 bread slice or 1 potato or $1 / 2$ bowl of cornflakes. All these products contain fiber. One should take 25-35 grams of fiber/day. Fiber rich diet
Volume 6 Issue 3 - 2017

\author{
Zainab Hussain \\ Diet Consultant in Foodservice setting at Tabba Heart \\ Institute(THI), Pakistan
}

\begin{abstract}
Correspondence: Zainab Hussain, Diet Consultant in Food service setting at Tabba Heart Institute(THI), Karachi, Pakistan, Email zainabhussain874@gmail.com
\end{abstract}

Received: February 23, 2017| Published: March 06, 2017

helps to prevent atherosclerosis and constipation. We should prefer whole fiber instead of refined fiber. Our diet should contain small amount of oil, sugar and sugary drinks altogether avoided. Deep fried items intake should be limited to once or twice a week. Use grilled, baked, steamed or shallow fried instead. Prefer polyunsaturated (corn oil, canola oil, sunflower oil) instead of saturated or trans fat (ghee, butter, margarine). Average intake of oil and sugar is 5-6 servings. One serving equals 1 tsp sugar; honey; oil; butter or 1 tsp dry fruits.

If diet doesn't contain each of the food group in equal amounts, the diet is considered to be incomplete and imbalanced. Such diet will not fulfil the body needs and consequently our body gets weakened.

\section{Acknowledgements}

I gratefully thank my boss Idris Mohammed Dawood who took out his precious time to edit this manuscript and bring in more clarity.

\section{Conflict of interest}

The author declares no conflict of interest. 